\section{Evaluation of antibiotic usage in swine reproduction farms in Umbria region based on the quantitative analysis of antimicrobial consumption}

\author{
Fausto Scoppetta, ${ }^{1}$ Marco Sensi, ${ }^{2}$ \\ Maria Pia Franciosini, ${ }^{3}$ \\ Marinella Capuccella ${ }^{1}$
}

${ }^{1}$ Umbria Regional Center of Veterinary

Pharmacovigilance, Institute for

Experimental Veterinary Medicine of

Umbria and Marche, Perugia; ${ }^{2}$ Institute

for Experimental Veterinary Medicine of

Umbria and Marche, Perugia;

${ }^{3}$ Department of Veterinary Medicine,

University of Perugia, Perugia, Italy

\begin{abstract}
Antibiotic use in food-producing animals has considerable impact on public health, especially with respect to the development and spread of antibiotic resistance. Pigs represent one of the main species in which antibiotics are frequently used for different purposes. Surveillance of antibiotic consumption and dose appropriateness, through novel approaches based on defined daily doses, is strongly needed to assess farms' antibiotic risk, in terms of spread of antibiotic resistance and possibile presence of residues in meat. In this study, antibiotic consumption was monitored in 14 swine reproduction farms, together with managerial, structural, and health aspects. Most of the controlled farms (65\%) were classified as at medium antibiotic risk, $21 \%$ at high antibiotic risk, and $14 \%$ at low antibiotic risk. Critical aspects of antibiotic administration concerned treatments for suckling and weaner piglets, oral antibiotic administration, treatment and diagnosis of gastroenteric infections, and use of critically important antimicrobials for human medicine, especially colistin. These aspects could be considered critical aspects of antibiotic use in from-farrow-to-wean/finish swine farms in the Umbria region and must be controlled to minimize risks. Even though a small number of farms in Umbria region are at high antibiotic risk, the risk of antibiotic resistance should be minimized, and management and biosecurity of the farms should be improved by extending the use of antimicrobial susceptibility tests and optimizing the diagnostic methods for infectious diseases. Furthermore, farmers' and veterinarians' knowledge of antibiotic resistance should be improved and the pru-
\end{abstract}

dent use of antibiotics encouraged to prevent the development and spread of resistant microorganisms.

\section{Introduction}

The transmission of antimicrobial resistant bacteria and/or antimicrobial resistance genes from animals to humans, although not fully investigated (Trauffler et al., 2014a, $2014 b$ ), exists and can be potentially responsible for therapeutic failures in treating infectious diseases in the human population (van der Fels-Klerx et al., 2011). Despite the European Union ban on the use of antibiotics as growth promoters in 2006, their use in therapy and/or prophylaxis /metaphylaxis still remains high in livestock production as reported by the European Surveillance of Veterinary Antimicrobial Consumption (ESVAC) project (EMA, 2016c). Italy is one of the countries where antimicrobial use in veterinary medicine is very high (EMA, 2016c). Although the ESVAC project represents an important international approach for the quantification of antibiotic use in veterinary medicine, different critical points, such as those related to the standardization of units of measurement and data sources, were found (Merle et al., 2014; Traufller et al., 2014a; EMA, 2016a). Recently, the European Medicine Agency (EMA) has proposed a new approach to quantify antibiotic usage in veterinary medicine, based on the concept of the defined daily dose (DDD), that is analogous to that in human medicine (EMA, 2016a; WHO, 2017). This represents a very innovative approach, overcoming the limits of ESVAC. It takes into consideration the strength of the antimicrobial class, which is fundamental to compare the consumption among antimicrobial classes.

The World Health Organization (WHO) has provided a list of critically important antimicrobials (CIAs) with the highest priority in human medicine. These drugs are characterized by elevated effectiveness; therefore low dosages might be sufficient to treat animals (Merle et al., 2014; EMA, 2016c). Third and fourth-generation cephalosporins, fluoroquinolones, macrolides, and, recently, colistin are the antimicrobial classes classified as CIAs by the WHO. Their use in veterinary medicine should be decreased and essentially, they would be prescribed after an antimicrobial susceptibility test has been performed (EMA，2016a，2016b，2016c，2016d; FAO/OIE/WHO, 2016). The surveillance of antimicrobial use is one of the suggested strategies for the better understanding of antibiotic consumption in livestock.
Correspondence: Fausto Scoppetta, Institute for Experimental Veterinary Medicine of Umbria and Marche, Umbria Regional Center of Veterinary Pharmacovigilance, via G. Salvemini, 1, 06126 Perugia, Italy.

Tel. +39753433006.

E-mail: f.scoppetta@izsum.it

Key words: Veterinary antimicrobial; Antibiotic use evaluation; Swine production; Antibiotic resistance; Veterinary pharmacoepidemiology.

Conflicts of interest: the authors declare no potential conflict of interest.

Acknowledgments: the authors gratefully acknowledge all the farmers for their participation, hospitality, and their availability to share their farm and antibiotic treatment data. Drs. Massimo Chiovoloni, Elide Colini, Marco Passini, Claudio Minciaroni, Stefano Mezzasoma, Patrizio Munzi, Maurizio Bordoni, Brigitta Favi, and Laura Mancini of Us1 Umbria 1 are acknowledged for their participation in the working group, questionnaire preparation and evaluation, farmer interviews, and farm inspections.

Received for publication: 6 July 2017. Revision received: 22 August 2017.

Accepted for publication: 24 August 2017.

This work is licensed under a Creative Commons Attribution-NonCommercial 4.0 International License (CC BY-NC 4.0).

CCopyright F. Scoppetta et al., 2017 Licensee PAGEPress, Italy

Italian Journal of Food Safety 2017; 6:6886

doi:10.4081/ijfs.2017.6886

Implementation of strategies for the reduction of their usage would consequently decrease the selection of antibiotic-resistant microorganisms (European Commission, 2015; Speksnijder et al., 2015; EMA, 2016a, 2016b; Lhermie et al., 2017). Therefore, reliable data on antibiotic consumption by food-producing species and single farms are fervently requested (Trauffler et al., 2014a). Antibiotic consumption is high in pig production, compared to other livestock. This is especially true during the first stage of the productive cycle where sows and piglets are often treated, frequently with CIAs, for therapeutic reasons and for prophylaxis and/or metaphylaxis (Merle et al., 2014). Risk analysis is an important tool to better understand and assess factors influencing a particular behaviour or necessity of antibiotics in food-producing animals (FAO, 2011; Teale and Moulin, 2012; EMA, 2015). Qualitative risk assessment is always performed at regional, national and international levels in 
the food-production chain (Landers et al., 2012), but it is based only on managerial, structural, and health variables. At the farm level, antibiotic risk should be considered as the probability that an irrational antibiotic use in livestock may have consequences on both human and veterinary public health. In this study, an evaluation of the qualitative use of antibiotics in the pig breeding farms of the Umbria region is proposed. It is based on reliable data on the use of antimicrobials and was evaluated with a DDD-based method, as suggested by EMA.

\section{Materials and Methods}

\section{Data collection and analysis}

All the breeding sow farms, with more than 60 reproductive females, were selected in the Umbria region (Central Italy). Data on type of farming and number of reared animals were collected from the national animal database (https://www.vetinfo.sanita.it/). Information about each farm, general characteristics, health status, and management were collected using both a questionnaire given to farmers and an analysis of the registers of treatments. The questionnaire was developed by a working group composed of public veterinarians, scientists, and experts in the veterinary drug and pig industry.

All the antibiotic administrations from $01 / 01 / 2016$ to $31 / 12 / 2016$ were evaluated and stored in a database using Microsoft Excel and Microsoft Access. The following information was collected for each antibiotic treatment: commercial name, dosage and duration of therapy with withdrawal periods, productive category, number and age of treated animals, and diagnosis. Data on the antimicrobials [active ingredients (a.i.) their strength, expressed in $\mathrm{mg} / \mathrm{g}$ or $\mathrm{mg} / \mathrm{mL}$; recommended daily dose for each productive category, expressed in $\mathrm{mg} / \mathrm{kg}$ body weight] were collected from the national veterinary drug handbook (https://www.vetinfo.sanita.it/j6_prontuario/public/)

Active ingredients were classified by their antimicrobial class according to the WHO indications (WHO, 2017). In particular, the analysis was performed taking into consideration all the antibiotics (AB) and CIAs. The monitoring was performed in all the individual farms that were investigated by productive categories and administration routes.

The estimation of $\mathrm{AB}$ and CIAs consumption was performed using the $D D D$ based approach, as suggested by the EMA (EMA, 2016a). It was evaluated using the following indicators: prescribed DDDs = total mgs of each a.i./provided EMA DDDvet value of this a.i.; DDDs/1000 animals-die $=$ [prescribed DDDs/(number of reared animals X day of observation (365)] $X 1000$; prevalence $(\%)=$ (number of treated animals/number of reared animals) $\mathrm{X}$ 100; DDDs/animals = prescribed DDDs/number of treated animals.

Prescribed DDDs and DDDs/1000 animals-die were used as the indicators for antibiotic consumption. Prevalence and DDDs/animals, respectively, were indicators of antibiotic exposition and intensity of antibiotic use. In addition, dosage appropriateness was evaluated for each a.i. as the ratio between the dose used and the does suggested by the drug leaflet, as suggested by Merle et al. (2014). Standard body weights used for dosage appropriateness were those provided by the EMA in the ESVAC project (EMA, 2016c).

Data collected from the questionnaire and from the antibiotic consumption and dose appropriateness analysis were used to perform the on-farm qualitative antibiotic use evaluation (Appendix).

The working group, which has built the questionnaire, provided a score from 0.5 to 3 for each used variable for the evaluation of antibiotic use, following the indications provided by different public authorities and authors (FAO, 2011; Italian Ministry of Health, 2012; Teale and Moulin, 2012; RUMA, 2013; EMA, 2015; FAO/OIE/WHO, 2016; EFSA, 2017). Assigned scores can be seen in Appendix.

For each considered farm, the antibiotic risk profile was evaluated by the sum of all the each variable assigned scores. The average score value was calculated and the farms were classified using the following system: farm score $\geq$ average score + standard deviation $(\sigma)$ : high antibiotic risk; farm score $<$ average score $+\sigma$ and $>$ average score $-\sigma$ : medium antibiotic risk; farm score $\leq$ average score value $-\sigma$ : low antibiotic risk.

\section{Statistical analysis}

Statistical analysis was performed using the software R 3.2.2. Simple logistic regression was used to determine if a high number of bred animals is correlated with a high antibiotic consumption. For this purpose, farms were split into a high and low number of reared animals, based on the average value of reared animals in all the farms investigated. Antibiotic consumption was evaluated as a relationship between DDDs/1000 animals-die calculated in each farm and DDDs/1000 animals-die evaluated in all the farms. Statistical analyses were performed to determine correlations among the inappropriateness of the dosage, use of
CIAs, administration route, and productive categories by evaluating the odds ratios (OR). The analyses were performed on each individual farm and on all the investigated farms collectively. Pearson's chi-squared test was used to determine statistical significance and $\mathrm{P}<0.05$ were considered significant.

\section{Results}

Eighteen from-farrow-to-wean/finish swine farms with $>60$ heads of breeding sows were identified in the Umbria region. Only 14 accepted to participate to the study. A total number of 36096 animals were reared in all the monitored farms during 2016. They were composed mostly of suckling piglets (SP) and weaners (W) $(64.96 \%)$, followed by sows and boars $(24.75 \%)$ and fattening-finishing pigs $(10.29 \%)$, bred only in five farms. The arithmetic mean of the reared animals in the 14 farms was 2578.29 (standard deviation $[\sigma]=1850.13$ ) and the $28.57 \%$ of the farms housed a higher than average number of animals. There were 2158 antibiotic treatments and $7215.58 \times 10^{4}$ prescribed DDDs. As to DDDs/1000 animals-die, 35.73\% of the farms registered a higher consumption compared with the total amount of DDDs/1000 animals-die (Table 1). The analysis of the CIAs highlighted a consumption of $2825.82 \times 10^{4}$ prescribed DDDs, corresponding to 2857.16 DDDs/1000 animals-die. CIAs prescribed using DDDs were $39.19 \%$ of the total prescribed DDDs. Four out of 14 farms (farms $1,2,8$ and 14) showed a higher consumption of CIAs (29\%) (Table 1). An odds ratio of 18 (IC95\% 1.51 - 498.33) was found between a high number of bred animals and high antibiotic consumption, although this was not statistically significant $(\mathrm{P}=0.078)$.

As reported in Table 1, penicillins, macrolides, tetracyclines, and polymyxins (colistin) were the most common antimicrobials used. Third-generation cephalosporins, penicillins, colistin and fluoroquinolones had the highest prevalence, while sulphonamides and trimethoprim, tetracyclines, and pleuromutilins had the highest intensity of use (DDDs/animals). Analysis of antibiotic consumption per classes and per farms can be seen in Table 1 .

The highest percentage of a.i. was related to SP-W, for both AB (58\%) and CIAs (78.34\%). The DDD-based analysis of consumption demonstrated the highest use in sows and boars, while the prevalence was high for SP-W for both total antibiotic consumption and CIAs (Table 2). A statistically significant association was found between 
the use of CIAs and SP-W $(\mathrm{OR}=5.08$, IC95\%: 4.26-6.07, $\mathrm{P}<2.2 \mathrm{e}^{-16}$ ).

Macrolides were the most common antimicrobials in sows and boars, followed by penicillins and tetracyclines. Polymyxins (colistin), penicillins, and tetracyclines were mostly used in SP-W (Table 2). Since it occurred in the majority of the farms $(71.43 \%)$, gastro-enteric diseases were the main reasons for antibiotic administration, followed by respiratory $(28.57 \%)$ and reproductive pathologies (14.29\%).

Antibiotics were mostly administered orally $(84.18 \%$ prescribed DDDs) in both SP-W (76.84\% prescribed DDDs) and sows and boars ( $88.07 \%$ prescribed DDDs). The oral route was the most used route. This also took into consideration, DDDs/1000 animals-die with 5894.28 DDDs/1000 animals-die compared to $1107.60 \mathrm{DDDs} / 1000$ animals-die used parenterally.

The highest percentage of treatments $(47.97 \%)$ were classified as correct dosage, $34.99 \%$ as under dosage, and $17.04 \%$ as over dosage (Figure 1); the correct dosage of CIAs administered was $39.67 \%$, while $45.64 \%$ were classified as under-dosage and $14.69 \%$ as over-dosage. $54.30 \%$ of parenteral treatments and the $33.45 \%$ of oral treatments were correctly administered. A statistically significant association was found between oral administration and under- or over-dosages (OR $=2.34$, IC $95 \%$ : $\left.1.98-2.77 ; P<2.2 \mathrm{e}^{-16}\right)$.

SP-W had the highest percentage of incorrect treatments $(62.25 \% \mathrm{AB}$ and $65.53 \%$ CIAs), most of which classified as under dosage $(49.67 \% \mathrm{AB}$ and $49.83 \%$ CIAs) (Figure 1). A significant association $\left(\mathrm{OR}=5.27\right.$; IC95\%: 4.46-6.24, $\left.P<2.2 \mathrm{e}^{-16}\right)$ was present between SP-W and incorrect dosage.

Statistically significant associations between dosage inappropriateness, use of CIAs and productive categories performed in each farms, evaluated as odds ratios, are listed in Table 3.

The $43 \%$ of the farms shows a statistically significant association with the probability of a mistaken antibiotic administration in terms of dosage (dosage inappropriateness) (Table 3 section A). Furthermore, $36 \%$ and the $21 \%$ of the farms displayed the same significant association for the treatment of SP-W and sows and boars, respectively (Table 3 section A).

Concerning the possible use of CIAs, $14 \%$ of the investigated farms exhibited a statistically significant association with the probability of administration of CIAs and $21 \%$ showed a significant association for the treatment of both SP-W and sows (Table 3 section $\mathrm{B}$ ).

Analysis of healthy and managerial

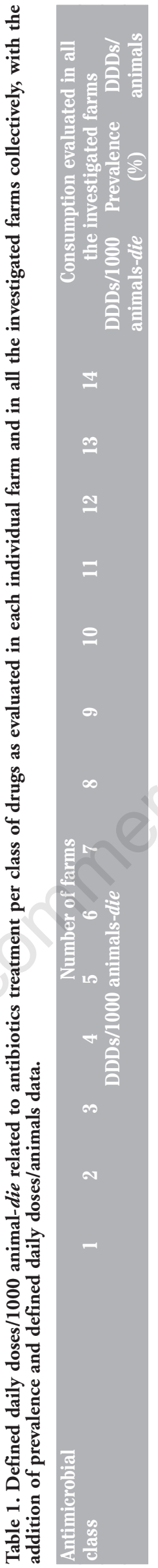

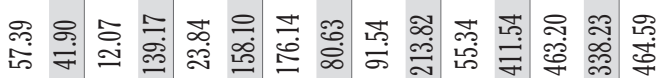

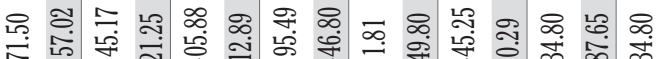

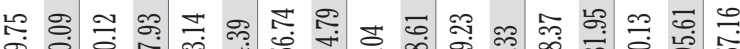

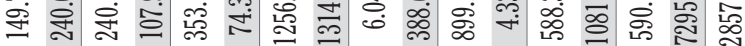

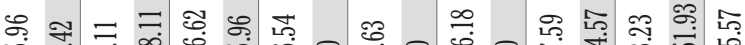

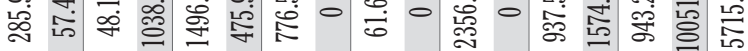

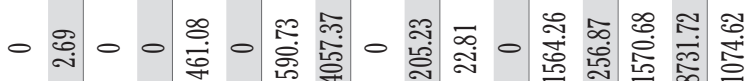

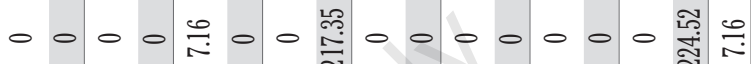

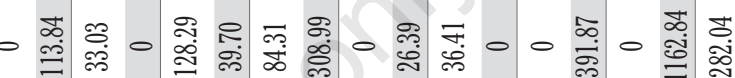

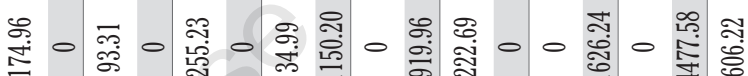

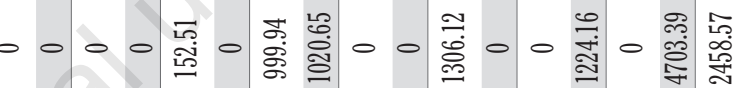

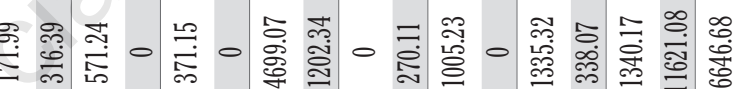

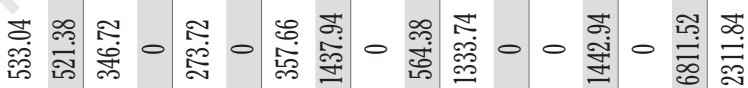
ब

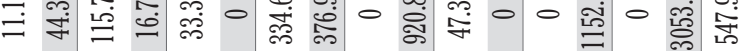
壳

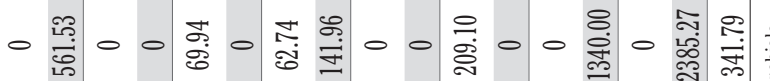

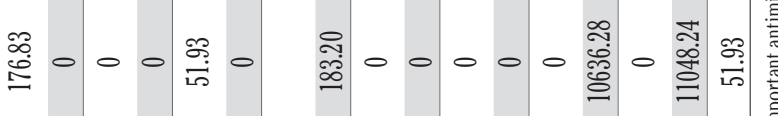

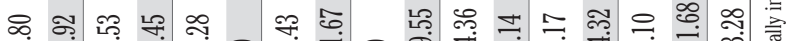

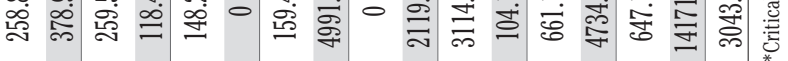

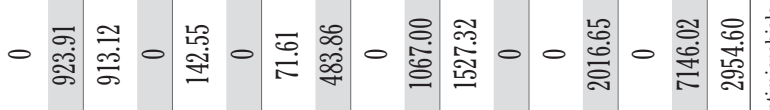

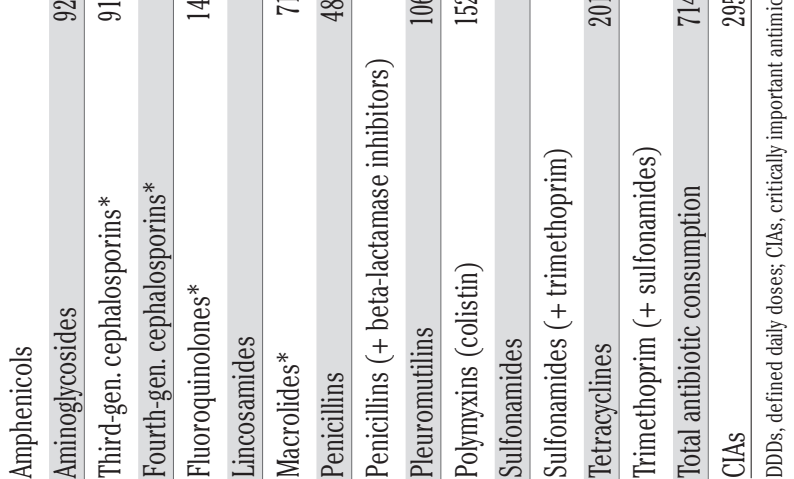


variables used for the evaluation of antibiotic use is summarized in Appendix. The open-cycle production system (weaners production) was applied to $71 \%$ of the farms. Recurrent infections, especially gastroenteritis $(71.43 \%)$, required antibiotic therapy in $57 \%$ of the 14 farms. Among these, in $29 \%$ of the holdings, the antibiotics were administered as therapy without any diagnosis, while the prophylactic or metaphylactic use of $\mathrm{AB}$ was documented in $50 \%$ of the farms.

The average score of antibiotic use was $30.96(\sigma=6.99)$. Three farms were classified at high antibiotic risk $(21 \%)$, while 9 $(65 \%)$ and $2(14 \%)$ were medium and low antibiotic risks, respectively.

\section{Discussion}

Our study represents the first Italian trial where antimicrobial consumption data were utilized for the evaluation of an antibiotic risk analysis and dosage appropriateness in pig breeding farms for implementing the new DDD-based approach, as suggested by the EMA. Our study was carried out on the effective consumption derived from the farm Register of Treatments and a specific questionnaire. This kind of approach made it possible to overcome some of limits caused by the evaluation of sale data, as reported in other national and international plans for antibiotic consumption (van der Fels-Klerx et al., 2011; Merle et al., 2012). Furthermore, it provides the possibility of a more economic antibiotic use evaluation by using data that already exists on the antibiotic use on farms, welfare, biosecurity, and structure/management.

Another advantage of our study was the antimicrobial consumption measurement units utilized. As already stated by EMA (EMA, 2016a), the antibiotic consumption analysis based on DDDs can overcome limits of other measurement units due to its basis on the antimicrobial strength and not on the standard animal weight. In our study, antibiotic consumption was expressed as DDDs/1000 animals-die, making it possible to weigh the data by the real number of reared animals. Such an approach could represent a suitable method to compare among productive categories of pigs, antibiotic classes, and farms. At the same time, it aims to be one of the elective units of measurement for analysing the relationship between antibiotic resistance and antibiotic usage in veterinary medicine (Collineau et al., 2016). Regarding productive categories, our results show a higher consumption of antibiotic in sows and boars, compared to $\mathrm{SP}-\mathrm{W}$. This is quite surprising, considering that SP-W were often the most frequently treated animals, as showed by other studies (Merle et al., 2014; van Rennings et al.,
2015). The use of a different unit of measurements in our study, could probably explain the highest antibiotic consumption in sows and boars, however further analysis should be done in the Umbria region and in Italy to assess which productive category is really the most treated in pig reproduction farms.

Dosage appropriateness is another major concern related to antibiotic use and the possible spread of antibiotic resistance (Ungemach et al., 2006; Merle et al., 2014). The possibility of selecting antibiotic-resistant bacteria after any antibiotic administration is common knowledge, especially in the case of a sub-therapeutic dosage (Barton, 2014). In this study, the use of the dosage inappropriateness ratio, expressed as a ratio between the used dose and the recommended dose from the leaflet, was another important item contributing to the antibiotic use evaluation in pig breeding farms. Since the differences between suggested doses are usually shown by different commercial products (i.e. dependent on disease severity or animal age) a $\pm 25 \%$ dose margin of correction range was adopted in order to minimize the bias, depending on the interpretation of the ratio (Timmerman et al., 2006). Our data revealed a correlation between inappropriate dosing and oral antibiotic administration, which could represent a risk of the increase in antibiotic resistance in pig production (Burow et al,
$\mathbf{A}$

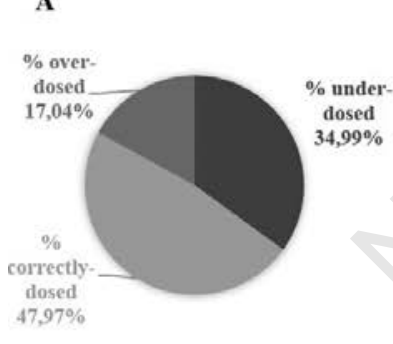

D

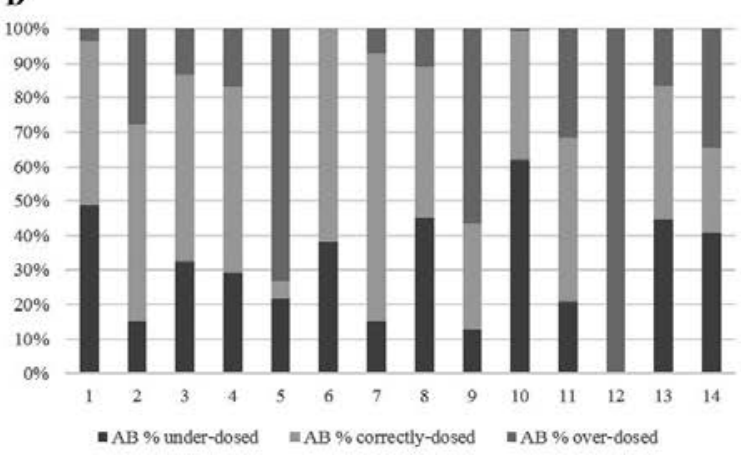

B

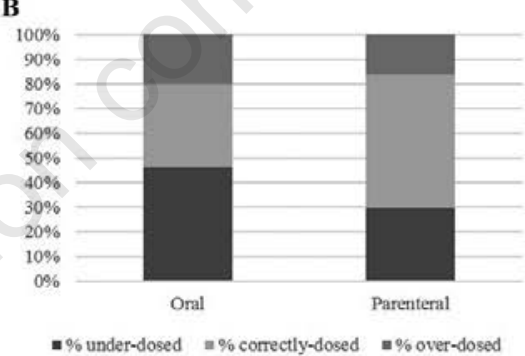

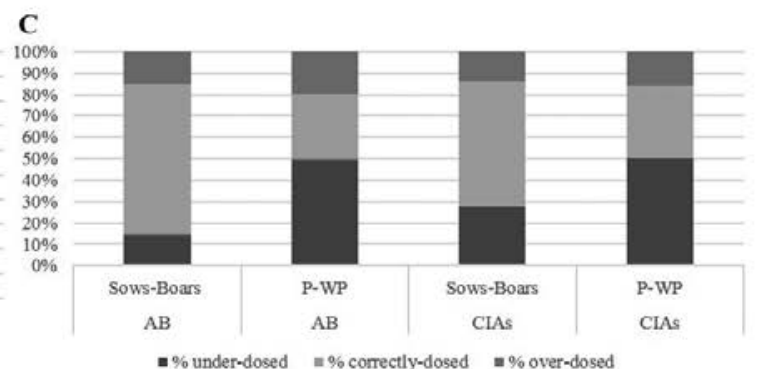

$\mathbf{E}$

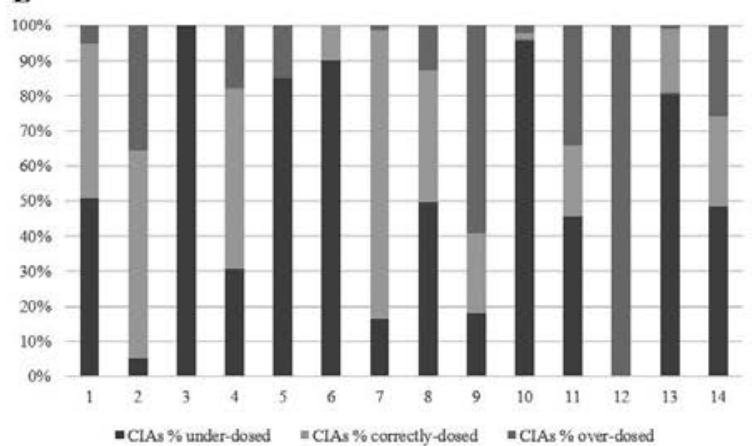

Figure 1. Analysis of appropriate dosing. A) total antimicrobial treatments, B) appropriateness per administration route, C) appropriateness per productive category, D) appropriateness of antibiotics in each individual farm, E) appropriateness of critically important antimicrobials in each individual farm. 
2014; Trauffler et al., 2014a). High percentages of under-dosed treatments administered orally were already reported by other studies (Callens et al., 2012; Merle et al., 2014). This percentage of inappropriateness can be due to the dosage expression (dose $/ \mathrm{kg}$ of feed or dose/litre of water) often used for orally administered group treatments of young animals (Timmerman et al., 2006). Therefore, it is necessary that clear recommendations with the correct dosage and for improvement of standard procedures for preparation and administration of antimicrobials should be made to both farmers and veterinarians. The tendency to perform oral group treatments in SP-W can partially explain the association between this productive category and inappropriate dosing. The fast growth rate in SP-W makes it difficult to identify an average body weight with which to calculate a suitable antibiotic dosage.

SP-W treatments can be also considered a critical issue in the association between the use of CIAs, especially colistin, and uncorrected dosages, which is responsible for the diffusion of antibiotic resistance (Trauffler et al, 2014a). In our work, colistin, which is mainly used in medicated feed, was widely used in SP-W, either as single a.i. or in association with other active agents, as seen by the evaluation of its prevalence. In pig production, it is wellknown that colistin is the first-choice antimicrobial for the treatment of gastroenteric infections, especially by $E$. coli, in SP-W (Timmerman et al., 2006; Callens et al., 2012; De Briyne et al., 2014). A significant reduction of its use in veterinary medicine is recommended because colistin could be one of the last resorts for treating multi-drug resistant infections in humans (EMA, 2016d). In our study, a high consumption of colistin was considered as suitable item for the evaluation of antibiotic risk at the farm level.

The most used antimicrobials emerging from our survey were similar to those reported in other countries (Callens et al., 2012; Merle et al., 2012; De Briyne et al., 2014; Merle et al., 2014; Trauffler et al., $2014,2014 b)$. The larger amount of prescribed products were broad-spectrum antibiotics, either as single a.i. (i.e. ampicillin) or in association with other a.i, i.e. amoxicillin plus colistin.

Macrolides were the most administered CIAs class, also showing high prevalence rates and DDDs/animals.

Due to the importance of macrolides for the treatment of campylobacteriosis infections in humans, their use should also be reduced in from-farrow-to-wean/finish pig farms in the Umbria region by improving vaccination and good management/hygiene practices.

In addition, it could be advisable to promote the use of other antimicrobial classes, such as pleuromutilins or tetracyclines, which have a similar spectrum of action and are not classified as CIAs by WHO (Timmerman et al., 2006).

In the investigated farms, the consumption of other CIAs antimicrobials (third and fourth- generation cephalosporins and fluoroquinolones) was limited. Moreover, they were often administered by the parenteral route, minimizing risk of under- or overdosing, as observed in another study carried out in north Europe (Timmerman et al., 2006; Trauffler et al., 2014a, 2014b).

Among the investigated farms, the use of antibiotics as prophylaxis/metaphylaxis was found in $29 \%$ of the farms and was not supported by antibiotic susceptibility tests. This protocol for antibiotic use must be reduced because such a misuse could increase the risk of antibiotic resistance spreading. At the same time, biosecurity and managerial improvements have to be carried out (Lhermie et al., 2017).

In our study, in order to evaluate the use of antibiotics, we also considered other aspects, such as structures, management, biosafety, vaccination programs, that might be useful for reducing antibiotic use in pig production (Laanen et al., 2013; Raith et al., 2016).

Among the 14 investigated farms, the all-in-all-out system was partially applied. Mainly due to the difficulties with its implementation in the traditionally shaped and

Table 2. Consumption of antibiotics and critically important antimicrobials in suckling piglets and weaners and sows and boars per classes of drugs.

\begin{tabular}{|c|c|c|c|c|c|c|c|c|}
\hline \multirow[b]{2}{*}{ Antimicrobial class } & \multicolumn{4}{|c|}{ SP-W } & \multicolumn{4}{|c|}{ Sows and boars } \\
\hline & $\begin{array}{c}\text { Prescribed } \\
\text { DDDs } \\
\left(\mathrm{x} 10^{4}\right)\end{array}$ & $\begin{array}{l}\text { DDDs/1000 } \\
\text { animals-die }\end{array}$ & $\begin{array}{c}\text { Prevalence } \\
(\%)\end{array}$ & $\begin{array}{l}\text { DDDs/ } \\
\text { animals }\end{array}$ & $\begin{array}{c}\text { Prescribed } \\
\text { DDDs } \\
\left(\mathrm{x} 10^{4}\right)\end{array}$ & $\begin{array}{l}\text { DDDs/1000 } \\
\text { animals-die }\end{array}$ & $\begin{array}{c}\text { Prevalence } \\
(\%)\end{array}$ & $\begin{array}{l}\text { DDDs/ } \\
\text { animals }\end{array}$ \\
\hline Amphenicols & 88.81 & 138.23 & 107.86 & 35.12 & 49.15 & 200.58 & 11.85 & 463.95 \\
\hline Aminoglycosides & 209.35 & 325.85 & 230.92 & 38.67 & 25.25 & 103.05 & 27.06 & 104.35 \\
\hline Third-gen. cephalosporins* & 237.07 & 368.99 & 839.15 & 12.05 & 0.42 & 1.70 & 0.11 & 416.67 \\
\hline Fourth-gen. cephalosporins* & 6.05 & 9.42 & 19.19 & 13.45 & 0 & 0 & 0 & 0 \\
\hline Fluoroquinolones* & 128.50 & 200.01 & 615.16 & 8.91 & 167.82 & 684.86 & 24.03 & 780.90 \\
\hline Lincosamides & 100.90 & 157.05 & 11.49 & 374.39 & 1.92 & 7.85 & 0.69 & 310.17 \\
\hline Macrolides* & 117.41 & 182.75 & 255.35 & 19.61 & 1095.14 & 4469.27 & 34.10 & 3590.63 \\
\hline Penicillins & 502.75 & 782.51 & 683.52 & 31.37 & 863.53 & 3524.07 & 119.03 & 811.25 \\
\hline Penicillins + beta-lactamase inhibitors & rs 5.98 & 9.30 & 2.78 & 91.54 & 0 & 0 & 0 & 0 \\
\hline Pleuromutilins & 95.58 & 148.77 & 44.14 & 92.35 & 288.77 & 1178.45 & 102.30 & 315.65 \\
\hline Polymyxins (colistin)* & 621.47 & 967.31 & 664.50 & 39.89 & 212.70 & 868.03 & 41.43 & 574.09 \\
\hline Sulfonamides & 0 & 0 & 0 & 0 & 4.08 & 16.65 & 1.04 & 438.71 \\
\hline Sulfonamides (+ trimethoprim) & 87.86 & 136.76 & 20.68 & 181.16 & 463.80 & 1892.77 & 75.29 & 688.85 \\
\hline Tetracyclines & 387.53 & 603.18 & 82.59 & 200.12 & 607.32 & 2478.46 & 146.12 & 464.76 \\
\hline Trimethoprim (+ sulfonamides) & 87.77 & 136.61 & 20.68 & 180.96 & 465.58 & 1900.01 & 75.29 & 691.48 \\
\hline Total antibiotic consumption & 2677.02 & 4166.73 & 3598.01 & 31.73 & 4245.47 & 17325.74 & 658.33 & 721.11 \\
\hline CIAs & 1110.50 & 1728.48 & 2393.36 & 47.70 & 1476.07 & 6023.86 & 99.68 & 4762.70 \\
\hline
\end{tabular}

SP-W, suckling piglets and weaners; DDDs, defined daily doses; CIAs, critically important antimicrobials. ${ }^{*}$ Critically important antimicrobials. 
continuous pig-flow system production farms. The lack of the adoption by the allin-all-out system could be partially replaced by suitable standardized procedures for cleaning/disinfection that would rotate the disinfectants in order to minimize the development of disinfectant-resistance (Postma et al., 2015).

Generally, the vaccination protocol adopted by each of the 14 farms was to address the improvement of animal health, mainly an improvement in the rates of gastro-enteric infections such as colibacillosis, in sows, suckling piglets and weaners. The appropriate implementation of suitable vaccination plans, combined with proper management, may play an important role in the reduction of antimicrobial use (Postma et al., 2015; Raith et al., 2016).
The variables used to determine statistically significant associations between the use of CIAs and inappropriate dosing provided a more accurate evaluation of antibiotic use the 14 farms. Farms 1, 6, and 7, followed by the same veterinarian, registered a negative association with inappropriate dosing. This means that the treated animals (in total or by productive categories) frequently received the correct antimicrobial dose. Alternatively, a positive association was seen at farms 8 and 13, both served by the same veterinarian, where the possibility of inadequate dosing was high. Concerning the treatment of breeding stock (sows and boars) with inappropriate doses, a negative association was seen in four out of the fourteen monitored farms. These results may indicate that adult animals are treated more accurately than the young ones, most likely due to a majority of individual and more precise treatments. However, some differences, must be reported farm by farm.

In summary, the results of our analysis on antibiotic risk showed that only 3 farms (21\%) out of the 14 are classified as at high risk, while the majority were into the two remaining categories $(65 \%$ medium risk and $14 \%$ low risk). These results suggest that high problematic from farrow to wean/fin$i s h$ pig farms in the Umbria region in terms of antimicrobial use are few, even if actions to improve management, biosecurity, diagnostic methods and farmers/veterinarians knowledge should be done to reduce the high risk and the medium risk farms.

Table 3. Statistically significant associations evaluated in each admitted farm between antibiotic treatments and inappropriate dosing (A) and use of critically important antimicrobials (B) performed on the total reared animals and on each productive category.

\begin{tabular}{|c|c|c|c|}
\hline Farm & Total reared animals & $\begin{array}{l}\text { A: Dosage inappropriateness } \\
\text { SP-W }\end{array}$ & Sows and boars \\
\hline 1 & - & - & OR: 0.30; IC95\%: 0.09-0.78; $\mathrm{P}=0.03$ \\
\hline 2 & OR: 0.63; IC95\%: $0.51-0.79 ; \mathrm{P}=4 \mathrm{e}-15$ & - & OR: $0.14 ;$ IC95\%: $0.07-0.25 ; \mathrm{P}=4.99 \mathrm{e}-12$ \\
\hline 3 & - & OR: 0.25; IC95\%: 0.09-0.64; $\mathrm{P}=0.005$ & OR: 2.99; IC95\%: 1.17-7.89;P=0.03 \\
\hline 4 & - & OR: 0.26; IC95\%: 0.14.046; $\mathrm{P}=2.46 \mathrm{e}-06$ & OR: 10.80; IC95\%: 2.76-71.11; $\mathrm{P}=0.0005$ \\
\hline 5 & OR: 17.65; IC95\%: 7.30-58.03; $\mathrm{P}=6 \mathrm{e}-14$ & & - \\
\hline 6 & OR: 0.54; IC95\%: 0.38-0.75; $\mathrm{P}=0.00037$ & - & OR: 0.52; IC95\%: 0.30-0.87; $\mathrm{P}=0.02$ \\
\hline 7 & OR: 0.22; IC95\%: 0.17-0.29; P<2.20e-16 & OR: 0.08; IC95\%: 0.05-0.11; P<2.20e-16 & - \\
\hline 8 & OR: 1.24; IC95\%: 1.05-1.47; P=0.011 & OR: 2.49; IC95\%: 1.90-3.30; P=6.22e-11 & OR: 0.74; IC95\%: 0.55-0.98; P=0.04 \\
\hline 9 & OR: 2.06; IC95\%: 1.06-4.24; P=0.05 & 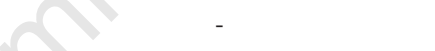 & - \\
\hline 10 & OR: 2.20; IC95\%: 1.55-3.18; P=1.37e-05 & OR: 13.76; IC95\%: 5.13-56.26; P=1.6e-08 & - \\
\hline 11 & - & OR: 5.53; IC95\%: 2.24-18.41; P=0.0004 & - \\
\hline 12 & - & - & - \\
\hline 13 & OR: 1.50; IC95\%: 1.15-1.95; P=0.002 & OR:8.97; IC95\%: 4.26-23.06; P=1.03e-09 & - \\
\hline 14 & OR: 2.90; IC95\%: 2.02-4.25; P=6.90e-9 & OR: 2.63; IC95\%: 4.26-23.06; $\mathrm{P}=0.001$ & OR: 5.20; IC95\%: $2.70-10.5 ; \mathrm{P}=3.23 \mathrm{e}-07$ \\
\hline Farm & Total reared animals & $\begin{array}{c}\text { B: CIAs use } \\
\text { SP-W }\end{array}$ & Sows \\
\hline 1 & OR: 5.08; IC95\%: 4.26-6.07; P<2.2e-16 & OR: 3.31; IC95\%: 2.18-5.21; P<2.34e-08 & - \\
\hline 2 & - & OR: 0.44; IC95\%: 0.33-0.59; P<4.26e-08 & OR: 3.34; IC95\%: 2.34-4.76; P=9.47e-12 \\
\hline 3 & OR: 0.45; IC95\%: $0.20-0.92 ; \mathrm{P}=0.05$ & OR: 0.14; IC95\%: 0.03-0.42; $\mathrm{P}=0.0006$ & - \\
\hline 4 & OR: 2.17; IC95\%: $1.32-3.62 ; \mathrm{P}=0.003$ & - & OR: 10.95; IC95\%: 3.71-39.81; P=2.27e-06 \\
\hline 5 & OR: 0.48; IC95\%: 0.28-0.79; P= 0.006 & OR: 0.13; IC95\%: 0.03-0.39; P=0.0003 & - \\
\hline 6 & - & OR: 5.38; IC95\%: 2.58-13.10; P=8.26e-06 & OR: 0.47; IC95\%: 0.24-0.87; P=0.03 \\
\hline 7 & - & - & OR: 0.54; IC95\%: 0.28-0.95; $\mathrm{P}=0.05$ \\
\hline 8 & OR: 0.83; IC95\%: 0.708-0.98; $\mathrm{P}=0.03$ & - & OR: 0.59; IC95\%: 0.42-0.82; P=0.002 \\
\hline 9 & - & - & - \\
\hline 10 & - & - & OR: 0.25; IC95\%: 0.08-0.63; P=0.08 \\
\hline 11 & - & - & - \\
\hline 12 & - & - & - \\
\hline 13 & - & OR: 4.18; IC95\%: 2.59-7.11; P=3.78e-09 & - \\
\hline 14 & - & OR: 0.63; IC95\%: 0.41-0.95; P=0.04 & OR: 5.04; IC95\%: 2.66-9.68; P=1.68e-07 \\
\hline
\end{tabular}

OR, odds ratio; IC, interval of confidence; SP-W, suckling piglets and weaners; CIAs, critically important antimicrobials. 


\section{Conclusions}

Several factors are involved with the decision to prescribe antibiotics in livestock and many quantitative and qualitative methods have been proposed to quantify antibiotic risk within farms (Landers et al., 2012). The majority of them are considered incomplete because they do not provide information on direct or indirect risks, which are the basics of risk classification.

Furthermore, these proposed approaches were essentially based on the personal evaluation of farm characteristics and were never applied to large-scale trials (Landers et al., 2012). Our study was able to enhance these critical points. It also represents the first approach in Italy where the data related to antibiotic use in pig farms were collected directly from the farm and utilized as a variable outcome in order to classify the antibiotic risk.

The use of antibiotics within pig breeding farms (from farrow to wean/finish) in the Umbria region should be strictly controlled because it could become a concern for the public health in terms of antibiotic resistance selection and possible presence of residues in pig productions. Therefore, even if the number of high risk farms is encouraging, an improvement in management and biosecurity and more responsible antibiotic treatment decisions should reduce the number of farms in the higher risk categories (high and medium categories). Furthermore, the use of antibiotic susceptibility tests should be encouraged and/or made compulsory to reduce the use of broad-spectrum antimicrobials, especially CIAs, and increase the use of narrow spectrum antibiotics.

The antibiotic treatment of suckling piglets-weaners, especially via the oral route, could be considered as an important critical issue and the accurate and strict control of oral administration may be necessary for minimizing the antibiotic risk.

\section{References}

Barton MD, 2014. Impact of antibiotic use in the swine industry. Curr Opin Microbiol 19:9-15.

Burow E, Simoneit C, Tenhagen BA, Käsbohrer A, 2014. Oral antimicrobials increase antimicrobial resistance in porcine E. coli-a systematic review. Prev Vet Med 113:364-75.

Callens B, Persoons D, Maes D, Laanen M, Postma M, Boyen F, Haesebrouck F, Butaye P, Catry B, Dewulf J, 2012. Prophylactic and metaphylactic antimi- crobial use in Belgian fattening pig herds. Prev Vet Med 106:53-62.

Collineau L, Belloc C, Stärk KD, Hémonic A, Postma M, Dewulf J, Chauvin C, 2016. Guidance on the Selection of Appropriate Indicators for Quantification of Antimicrobial Usage in Humans and Animals. Zoon Publ Health 64:165-84.

De Briyne N, Atkinson J, Pokludová L, Borriello SP, 2014. Antibiotics used most commonly to treat animals in Europe. Vet Rec 175:325.

EFSA, 2017. EMA and EFSA Joint Scientific Opinion on measures to reduce the need to use antimicrobial agents in animal husbandry in the European Union, and the resulting impacts on food safety (RONAFA). EFSA J 15:4666.

EMA, 2015. Guideline on the assessment of the risk to public health from antimicrobial resistance due to the use of an antimicrobial veterinary medicinal product in food producing animals. Draft. EMA/CVMP/AWP/706442/ 2013. Committee for Medicinal Products for Veterinary Use (CVMP) Codex Alimentarius Commission. European Medicines Agency, London, UK.

EMA, 2016a. Defined daily doses for animals ( DDDvet) and defined course doses for animals (DCDvet) European Surveillance of Veterinary Antimicrobial Consumption (ESVAC). EMA/224954/2016 Veterinary Medicines Division. European Medicines Agency, London, UK.

EMA, 2016b. ESVAC: Vision, Strategy and Objectives 2016-2020. European Surveillance of Veterinary Antimicrobial Consumption. EMA/326299/2015 Veterinary Medicines Division. European Medicines Agency, London, UK.

EMA, 2016c. Sales of veterinary antimicrobial agents in 29 European countries in 2014. Trends from 2011 to 2014 Sixth ESVAC report 14 October 2016 EMA/61769/2016 Veterinary Medicines Division. European Medicines Agency, London, UK.

EMA, 2016d. Updated advice on the use of colistin products in animals with in the European Union: development of resistance and possible impact on human and animal health. EMA /CVMP/CHMP/ 231573/2016. Committee for Medicinal Products for Veterinary use (CVMP) Committee for Medicinal Products for Human Use (CHMP). European Medicines Agency, London, UK.
European Commission, 2015. Notice of European Commission 2015/C 299/04. Guidelines for the prudent use of antimicrobials in veterinary medicine (2015/C 299/04). In: Official Journal of the European Union. European Commission, Bruxelles, Belgium.

FAO, 2011. Guidelines for risk analysis of foodborne antimicrobial resistance. CAC/GL 77-2011.

FAO/OIE/WHO, 2016. Antimicrobial resistance. A manual for developing national action plans. FAO, Rome, Italy.

Italian Ministry of Health, Department of Health public veterinary, food safety and the governing bodies for the protection of health, 2012. Manual - Biosafety and correct and rational use of antibiotics in animal husbandry. Italian Ministry of Health, Rome, Italy.

Laanen M, Persoons D, Ribbens S, de Jong E, Callens B, Strubbe M, Maes D, Dewulf J, 2013. Relationship between biosecurity and production/antimicrobial treatment characteristics in pig herds. Vet J 198:508-12.

Landers TF, Cohen B, Wittum TE, Larson EL, 2012. A review of antibiotic use in food animals: perspective, policy, and potential. Public Health Rep 127:4-22.

Lhermie G, Gröhn YT, Raboisson D, 2017. Addressing antimicrobial resistance: an overview of priority actions to prevent suboptimal antimicrobial use in foodanimal production. Front Microbiol 7:2114.

Merle R, Hajek P, Käsbohrer A, HeggerGravenhorst C, Mollenhauer Y, Robanus M, Ungemach FR, Kreienbrock L, 2012. Monitoring of antibiotic consumption in livestock: a German feasibility study. Prev Vet Med 104:34-43.

Merle R, Robanus M, Hegger-Gravenhorst C, Mollenhauer Y, Hajek P, Käsbohrer A, Honscha W, Kreienbrock L, 2014. Feasibility study of veterinary antibiotic consumption in Germany-comparison of ADDs and UDDs by animal production type, antimicrobial class and indication. BMC Vet Res 10:7.

Postma M, Stärk KD, Sjölund M, Backhans A, Beilage EG, Lösken S, Belloc C, Collineau L, Iten D, Visschers V, Nielsen EO, Dewulf J, MINAPIG consortium, 2015. Alternatives to the use of antimicrobial agents in pig production: a multi-country expert-ranking of perceived effectiveness, feasibility and return on investment. Prev Vet Med 118:457-66.

Raith J, Trauffler M, Firth CL, Lebl K, Schleicher C, Köfer J, 2016. Influence of porcine circovirus type 2 vaccination 
on the level of antimicrobial consumption on 65 Austrian pig farms. Vet Rec 178:504.

RUMA, 2013. Responsible use of antimicrobials in pig production. Responsible Use Of Antimicrobials In Agriculture Alliance, London, UK.

Speksnijder DC, Mevius DJ, Bruschke CJ, Wagenaar JA, 2015. Reduction of veterinary antimicrobial use in the Netherlands. The Dutch success model. Zoonoses Publ Health 62:79-87.

Teale CJ, Moulin G, 2012. Prudent use guidelines: a review of existing veterinary guidelines. Rev Sci Tech Off Int Epiz 31:343-54.

Timmerman T, Dewulf J, Catry B, Feyen B, Opsomer G, de Kruif A, Maes D, 2006. Quantification and evaluation of antimi- crobial drug use in group treatments for fattening pigs in Belgium. Prev Vet Med 16:251-63.

Trauffler M, Griesbacher A, Fuchs K, Köfer J, 2014a. Antimicrobial drug use in Austrian pig farms: plausibility check of electronic on-farm records and estimation of consumption. Vet Rec 175:402.

Trauffler M, Obritzhauser W, Raith J, Fuchs K, Köfer J, 2014b. The use of the "highest priority critically important antimicrobials" in 75 Austrian pig farmsevaluation of on-farm drug application data. Berl Munch Tierarztl Wochenschr 127:375-83.

Ungemach FR, Müller-Bahrdt D, Abraham G, 2006. Guidelines for prudent use of antimicrobials and their implications on antibiotic usage in veterinary medicine. Int J Med Microbiol 296:33-8.

van der Fels-Klerx HJ, Puister-Jansen LF, van Asselt ED, Burgers SL, 2011. Farm factors associated with the use of antibiotics in pig production. J Anim Sci 89:1922-9.

van Rennings L, von Münchhausen C, Ottilie H, Hartmann M, Merle R, Honscha W, Käsbohrer A, Kreienbrock L, 2015. Cross-sectional study on antibiotic usage in pigs in Germany. PLoS One 18:10.

WHO, 2017. Critically important antimicrobials for human medicine. 5th Revision 2016. WHO Advisory Group on Integrated Surveillance of Antimicrobial Resistance (AGISAR). WHO, Geneva, Switzerland. 